Wir haben uns daran gewöhnt: Bevor der Operationstermin angesetzt wird, muss der informierte Patient dem Eingriff frei zugestimmt haben. Doch damit ist es nicht getan. Wasserfallen und Simon zeigen mit ihrer Studie aus dem CHUV, dass der Gesetzgeber auf Bundes- oder auch Kantonsebene für immer mehr Fragen im Spitalkontext eine explizite Zustimmung des Patienten verlangt. Rechnungsstellung an die Krankenkasse und Rolle des Vertrauensarztes, Umgang mit Gewebsproben, Benützung der KG für die Forschung, Mitwirkung des Patienten im Unterricht. Ungelöst sind zwei Aspekte:

Ressourcen: Entweder werden diese Fragen beim Spitaleintritt wie blosse Formalitäten abgehakt - oder aber die Diskussion braucht Zeit und damit Geld. Doch wie wichtig sind diese Fragen wirklich, wenn die dafür nötigen Ausgaben mit den Kosten nötiger Operationen, aber auch mit den Ausgaben für eine gute Volksschule, für Sicherheit usw. konkurrieren?
Begrenzte Spannkraft des Patienten: Viele Patienten im Spital sind aufgrund ihres Alters oder krankheitsbedingter Schwäche kaum in der Lage, der medizinischen Behandlung bewusst zuzustimmen. Ihnen eine ganze Reihe von weiteren Fragen zum ausdrücklichen Entscheid vorzulegen, erweist sich aufgrund der Studie als faktisch nicht machbar. Das Ganze wird zur Alibiübung.

Diese Erkenntnis ist politisch (noch?) nicht korrekt. Aber wir sollten dennoch darüber nachdenken. Persönlich denke ich, dass für medizinische Studien Ethikkommissionen und für viele andere Fragen möglichst breit getragene Patientenorganisationen als Ansprechpartner des Spitals viele Lösungen legitimieren könnten, mit denen sich die meisten Spitalpatienten selbst nicht wirklich kritisch auseinandersetzen können.

Hanspeter Kuhn, Fürsprecher, stv. Generalsekretär FMH

\title{
Elicitation d'un consentement groupé auprès de patients hospitalisés: double étude pilote
}

\section{Jean-Blaise Wasserfallen ${ }^{a}$, Jeanne-Pascale Simon ${ }^{b}$ \\ a Direction médicale, Centre Hospitalier Universitaire Vaudois (CHUV), Lausanne \\ b Unité des affaires juridiques Centre Hospitalier Universitaire Vaudois (CHUV), Lausanne}

\section{Remerciements}

Les auteurs remercient Mesdames Katia Siciliano, Nathalie Villers, Corinne Visomblien, et Monsieur François Schopfer pour leur travail de recueil de consentement auprès des patients.

Correspondance:

Dr Jean-Blaise Wasserfallen Direction médicale

Centre Hospitalier Universitaire Vaudois

Rue du Bugnon 46

CH-1011 Lausanne

Tél. 0213141802

Fax 0213141818

jbw@chuv.ch

\section{Contexte}

Les progrès technologiques en médecine ont abouti à une spécialisation de plus en plus poussée des professionnels de la santé, surtout en hôpital. Il en résulte que les prises en charge deviennent de plus en plus complexes et pluridisciplinaires, nécessitant la transmission précise d'informations entre les différents professionnels impliqués pour garantir un résultat optimum et minimiser le risque d'effets secondaires des procédures diagnostiques et thérapeutiques. Par ailleurs, la recherche médicale et l'enseignement sont des missions fondamentales des hôpitaux universitaires, dont l'enjeu d'intérêt collectif n'est plus à démontrer. Pour développer ces activités, les patients et le matériel important récolté dans le cadre d'un hôpital universitaire doivent pouvoir être mis à disposition des enseignants et des chercheurs, ceci dans le respect des droits des patients et aux conditions prévues par le droit d'une part et dans un environnement de concurrence internationale constante d'autre part.

Parallèlement à ces difficultés pratiques, le cadre légal devient de plus en plus contraignant, les différentes lois concernées poursuivant parfois des buts divergents. Si le Code Pénal suisse (CP) [1] punit toute procédure diagnostique ou thérapeutique invasive menée sans l'obtention d'un consentement de la personne concernée, de même que la violation du secret professionnel (CP art. 321), la Loi sur la santé Publique Vaudoise (LSP) [2] exige explicitement que le patient donne son consentement au traitement et à la transmission de données sensibles (LSP art. 23 et 80). Finalement, la LSP demande que l'hôpital constitue et conserve un dossier sur chaque patient (LSP art. 87), et la Loi sur l'Assurance Maladie (LAMal art. 42) [3] que l'hôpital remette à l'assurance une facture détaillée et compréhensible, contenant de ce fait un code diagnostic protégé par principe par le secret professionnel. L'article 42 LAMal prévoit en même temps le droit pour le patient d'exiger que ces données médicales ne soient transmises qu'au médecin-conseil de l'assurance, lui-même soumis au secret professionnel. Enfin, concernant la recherche, la LSP (LSP art. 23 in fine) prévoit qu'un échantillon biologique ne peut être utilisé qu'aux fins approuvées par la personne concernée, et le CP punit l'accès à des données protégées par le secret professionnel sans l'autorisation du patient (CP art. 321bis).

Pour satisfaire à ces multiples obligations et respecter le droit du patient à l'autodétermination, le Centre Hospitalier Universitaire Vaudois (CHUV) a imaginé fournir au patient une information simultanée sur le traitement des données cliniques, l'utilisation de ces données et des échantillons biologiques pour la recherche, la participation aux activités d'enseignement, ainsi que la transmission des informations aux assureurs, et recueillir son consentement groupé par écrit à son admission ou au plus tard pendant son séjour hospitalier. Le lieu idéal pour fournir cette information n'est en effet pas facile à trouver, compte tenu des multiples voies d'admission des patients. A noter que cette démarche est complémentaire au recueil de consentement nécessaire (et non remis en question) pour toute procédure diagnostique ou thérapeutique invasive.

Comme, d'autre part, les patients hospitalisés sont plus ou moins gravement atteints dans leur santé phy- 
sique et psychique (au point que leur capacité de discernement est parfois altérée), et avant tout préoccupés de recevoir des soins et pas tellement de remplir des démarches administratives, il paraissait raisonnable d'anticiper certaines difficultés à appliquer cette démarche. C'est la raison pour laquelle il a été décidé d'effectuer deux phases test de trois mois chacune: l'une sur deux services d'hospitalisation (l'un médical et l'autre chirurgical), et l'autre en consultation de préhospitalisation chirurgicale, par où passe la majorité des patients convoqués pour une hospitalisation liée à une intervention chirurgicale.

\section{Matériel et méthode}

Une feuille d'information a été développée pour couvrir l'ensemble des exigences légales liées à un consentement groupé. Elle porte sur le traitement des données personnelles et la constitution d'un dossier hospitalier, la participation aux activités d'enseignement et de recherche et la méthode de facturation des séjours. Cette feuille d'information se présente sous forme d'une brochure (en fichier PDF sur Internet à l'adresse www.saez.ch $\rightarrow$ Numéro actuel où Archives $\rightarrow 2009 \rightarrow 6$ ).

Elle est envoyée avec les papiers d'admission pour les patients convoqués, et distribuée avec la documentation d'information dans les étages pour les patients admis en urgence. Les infirmières la remettent directement aux patients en consultation de pré-hospitalisation chirurgicale. Elle est accompagnée d'un formulaire permettant de recueillir le consentement explicite du patient, ou au contraire les limitations qu'il souhaiterait apporter à son consentement (figure 1).

Figure 1

Feuille d'information aux patients.

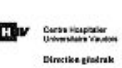

étiquette

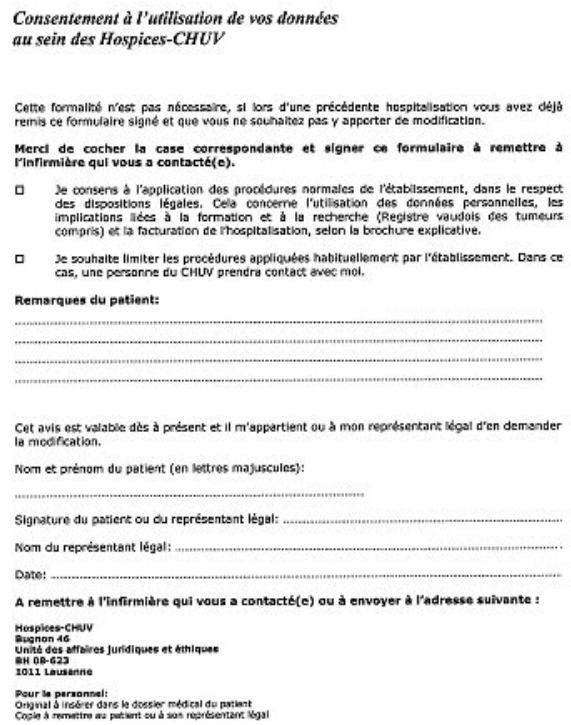

|||||||||||||||||||||||||||||

\section{Einholen einer Zustimmungs-} erklärung bei hospitalisierten

\section{Patienten: doppelte Pilotstudie}

Kontext: Die schweizerische Gesetzgebung und Gerichtspraxis verlangen immer häufiger eine ausdrückliche Zustimmung des Patienten zu einer Vielzahl von Situationen, die im Verlauf eines Spitalaufenthalts auftreten können. Es kann schwierig und kostspielig sein, all diesen Anforderungen nachzukommen. Das CHUV hat den Versuch unternommen, die Implikationen der rechtlichen Anforderungen für die Praxis zu überprüfen.

Methode: Die Untersuchung fand in zwei Spital-Abteilungen (internistisch und chirurgisch) statt und auch in der chirurgischen Sprechstunde vor dem Spitaleintritt. Während zweimal drei Monaten haben ausgewählte Krankenpflegerinnen Informationsblätter verteilt, die alle rechtlichen Vorschriften zum allgemeinen Einverständnis zusammenfassten, und anschliessend die Zustimmungserklärung, bzw. die von Patienten gewünschten Einschränkungen dieser Zustimmungserklärung, eingeholt.

Ergebnisse: In den beiden Spital-Abteilungen haben nur 322 (33\%) von 983 anwesenden Patienten eine Zustimmungserklärung abgegeben, von denen $94 \%$ diese ohne Einschränkung erteilt haben. Bei $661 \mathrm{~Pa}$ tienten konnte eine Zustimmung trotz wiederholter Besuche nicht eingeholt werden. Von diesen waren $\mathbf{4 2} \%$ nicht in der gesundheitlichen Verfassung, um zu antworten, $30 \%$ haben eine Zustimmung abgelehnt, $\mathbf{2 1} \%$ sind ausgetreten oder wurden verlegt, bevor sie zustimmen konnten, und $7 \%$ sprachen eine Sprache, in die eine Übersetzung nicht geleistet werden konnte. In die Sprechstunde vor Spitaleintritt kamen $855 \mathrm{~Pa}$ tienten, von denen 756 (88\%) von den Krankenpflegerinnen kontaktiert werden konnten. 589 Zustimmungserklärungen (78\% der erfolgreich kontaktierten Patienten, 69\% aller Patienten) konnten eingeholt werden, $96 \%$ davon ohne Einschränkungen.

Schlussfolgerung: Das Einholen einer expliziten Zustimmungserklärung bei hospitalisierten Patienten bzw. in einer Sprechstunde vor Spitaleintritt ist nur in einem variablen Prozentsatz von Fällen möglich und erfordert - bei einer niedrigen Anzahl von Verweigerungen - einen bedeutenden Zeit- und Personalaufwand. Es müssen andere Lösungen gesucht werden, um den aktuellen rechtlichen Anforderungen zu genügen. 


\section{Services d'hospitalisation}

Une infirmière visite systématiquement les patients pour répondre aux questions qu'aurait pu susciter la feuille d'information, fournir les informations supplémentaires, si nécessaire, et recueillir le consentement et ses limites selon les cas. Une fois celui-ci obtenu, elle entre la réponse dans le système d'information et transmet aux partenaires impliqués dans la prise en charge une éventuelle limitation du consentement. Elle classe également l'original de la feuille de consentement dans le dossier du patient, alors qu'une copie lui en est donnée.

Le test a été réalisé pendant 3 mois dans deux services: le Service de Médecine interne, de 150 lits, dont

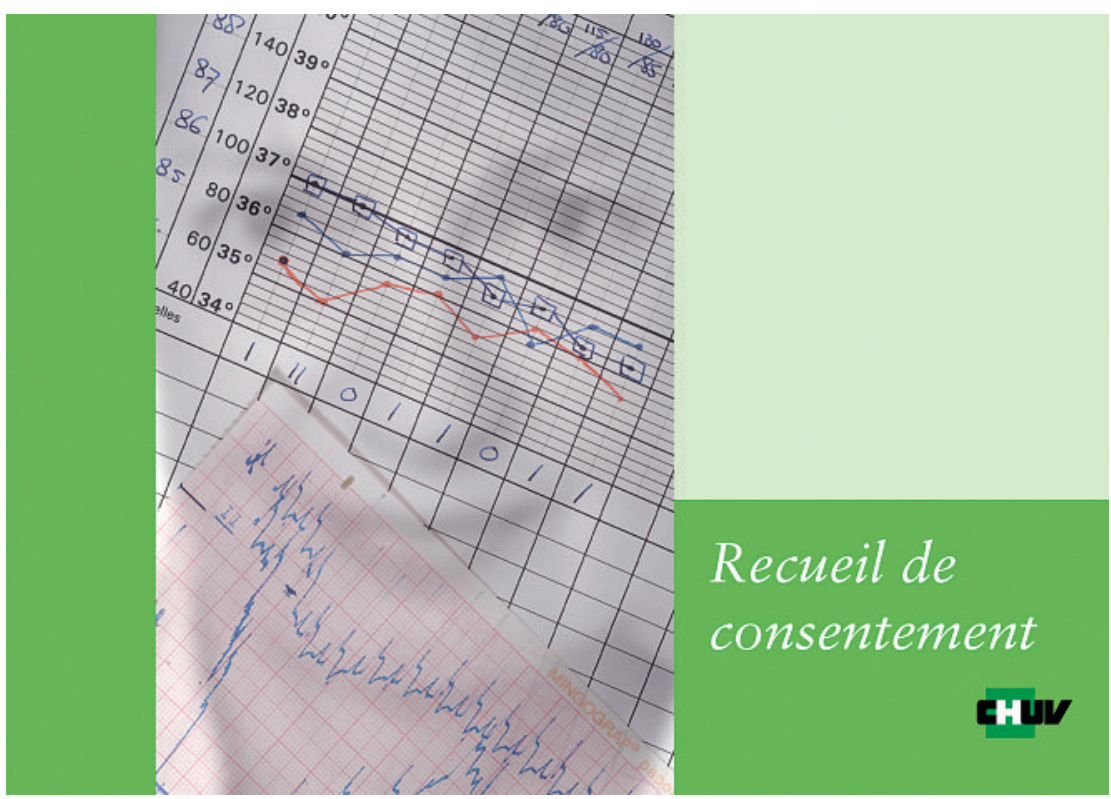

Une feuille d'information résumant l'ensemble des exigences légales pour un consentement groupé était distribuée. *

* Vous trouverez la feuille de recueil de consentement avec tous les textes sur Internet sous www.bullmed.ch $\rightarrow$ archives $2010 \rightarrow 8$ à la fin de l'article

Tableau 1

Distribution des résultats par service d'hospitalisation.

\begin{tabular}{|c|c|c|c|c|c|c|}
\hline & \multicolumn{2}{|c|}{ Médecine interne } & \multicolumn{2}{|c|}{ Oto-rhino-laryngologie } & \multirow{2}{*}{$\begin{array}{l}\text { Total } \\
\text { Nombre }\end{array}$} & \multirow[b]{2}{*}{$\%$} \\
\hline & Nombre & $\%$ & Nombre & $\%$ & & \\
\hline Patients & 683 & 69,5 & 300 & 30,5 & 983 & 100,0 \\
\hline $\begin{array}{l}\text { Consentements } \\
\text { obtenus }\end{array}$ & 220 & 32,2 & 102 & 34,0 & 322 & 32,8 \\
\hline $\begin{array}{l}\text { Consentements } \\
\text { complets }\end{array}$ & 205 & 93,2 & 97 & 95,1 & 302 & 93,8 \\
\hline $\begin{array}{l}\text { Limitation données } \\
\text { personnelles }\end{array}$ & 1 & 0,4 & 0 & 0,0 & 1 & 0,3 \\
\hline Limitation formation & 12 & 5,5 & 5 & 4,9 & 17 & 5,3 \\
\hline Limitation recherche & 0 & 0,0 & 0 & 0,0 & 0 & 0,0 \\
\hline $\begin{array}{l}\text { Limitation facturation } \\
\text { APDRG }\end{array}$ & 2 & 0,9 & 0 & 0,0 & 2 & 0,6 \\
\hline $\begin{array}{l}\text { Consentements } \\
\text { non obtenus }\end{array}$ & 463 & 67,8 & 198 & 66,0 & 661 & 67,2 \\
\hline
\end{tabular}

la majorité des patients est hospitalisée sur le mode urgent, et le Service d'Oto-rhino-laryngologie, de 30 lits, dont les patients sont pour la plupart convoqués.

\section{Consultation au Centre de pré-hospitalisation chirurgicale (CPC)}

Le test a aussi été réalisé pendant 3 mois, dans la salle d'attente du CPC. Une équipe de trois infirmières à temps partiel couvre la tranche horaire de 8 heures à 17 heures, un patient étant convoqué toutes les 30 minutes le matin et deux l'après-midi.

\section{Analyse des résultats}

Pour les deux essais pilote, les résultats ont été catalogués et analysés en fonction de la réponse obtenue ou du type de limitation rencontré. Une enquête qualitative sur l'accueil de la démarche auprès des patients et des soignants a aussi été réalisée.

\section{Résultats}

\section{Services d'hospitalisation}

983 patients ont été hospitalisés pendant les trois mois de la phase test et 322 patients ont fourni un consentement (33\% des patients): 302 consentements étaient non limités (94\%), 1 limité pour le recueil des données personnelles, 17 pour la collaboration à la formation, et 2 pour la transmission des données de facturation à la caisse maladie. Aucune limitation n'a été demandée pour la recherche. La distribution des réponses en fonction du type de service figure au tableau 1.

Chez 661 patients (67\%), le consentement n'a pas pu être recueilli, malgré des visites répétées: $42 \%$ de ces patients n'étaient pas en état de répondre à cause de leur affection physique ou psychique (hospitalisation en soins continus, phase pré- ou post-opératoire, incapacité de discernement, âge avancé, confusion, problème physique de type malvoyance ou surdité); 30\% d'entre eux ont refusé d'entrer en matière ou de remplir le formulaire, arguant du fait qu'ils n'étaient pas intéressés à la démarche, saturés par l'information reçue avant et pendant l'hospitalisation. Certains étaient d'accord oralement mais refusaient de signer. Finalement, $21 \%$ des patients étaient sortis ou transférés sans avoir rendu le formulaire et $7 \%$ parlaient des langues étrangères qui rendaient le recueil du consentement particulièrement difficile.

Malgré ces résultats mitigés, la démarche était de manière générale bien accueillie et par les patients et par les équipes soignantes. Les infirmières chargées de la démarche ont noté que de manière générale, l'information écrite était insuffisante et qu'il était indispensable de donner des explications orales supplémentaires. De plus, des passages répétés étaient nécessaires, en moyenne 5 par patient. Le temps consacré aux explications était plus important en Médecine qu'en ORL, les patients souhaitant pouvoir s'en référer à un proche avant de signer le formulaire de consentement. L'envoi des documents à domicile s'avérait inu- 
Tableau 2

Distribution des résultats au Centre de Pré-hospitalisation Chirurgicale (CPC), par mois.

\begin{tabular}{|c|c|c|c|c|c|c|c|c|}
\hline & $\begin{array}{l}\text { Mois } 1 \\
\text { Nombre }\end{array}$ & $\%$ & $\begin{array}{l}\text { Mois } 2 \\
\text { Nombre }\end{array}$ & $\%$ & $\begin{array}{l}\text { Mois } 3 \\
\text { Nombre }\end{array}$ & $\%$ & $\begin{array}{l}\text { Total } \\
\text { Nombre }\end{array}$ & \\
\hline $\begin{array}{l}\text { Patients venus } \\
\text { au CPC }\end{array}$ & 252 & 29,5 & 318 & 37,2 & 285 & 33,3 & 855 & 100,0 \\
\hline $\begin{array}{l}\text { Patients vus pour } \\
\text { consentement } \\
\text { groupé }\end{array}$ & 209 & 82,9 & 293 & 92,1 & 254 & 89,1 & 756 & 88,4 \\
\hline $\begin{array}{l}\text { Consentements } \\
\text { obtenus }\end{array}$ & 171 & 81,8 & 223 & 76,1 & 195 & 76,8 & 589 & 77,9 \\
\hline $\begin{array}{l}\text { Consentements } \\
\text { complets }\end{array}$ & 168 & 98,2 & 211 & 94,6 & 187 & 95,9 & 566 & 96,1 \\
\hline $\begin{array}{l}\text { Limitation } \\
\text { données } \\
\text { personnelles }\end{array}$ & 1 & 0,5 & 1 & 0,4 & 0 & 0,0 & 2 & 0,3 \\
\hline $\begin{array}{l}\text { Limitation } \\
\text { formation }\end{array}$ & 1 & 0,5 & 7 & 3,1 & 3 & 1,5 & 11 & 1,9 \\
\hline $\begin{array}{l}\text { Limitation } \\
\text { recherche }\end{array}$ & 0 & 0,0 & 5 & 2,2 & 3 & 1,5 & 8 & 1,4 \\
\hline $\begin{array}{l}\text { Limitation } \\
\text { facturation } \\
\text { APDRG }\end{array}$ & 1 & 0,5 & 3 & 1,3 & 2 & 1,0 & 6 & 1,0 \\
\hline $\begin{array}{l}\text { Consentements } \\
\text { non obtenus }\end{array}$ & 38 & 18,2 & 70 & 23,9 & 59 & 23,2 & 167 & 22,1 \\
\hline
\end{tabular}

tile, les patients ne les apportant pas lors de leur hospitalisation, lorsqu'ils en avaient pris connaissance.

\section{Centre de pré-hospitalisation chirurgicale}

855 patients ont consulté le CPC pendant les 3 mois de la phase test et 756 patients ( $88 \%$ ) ont été vus pour le recueil du consentement groupé. Sur ces derniers, $78 \%$ (589 patients) ont fourni un consentement (69\% du total des patients): 566 consentements étaient complets (96\%), 11 limités pour la collaboration à la formation $(1,9 \%), 8$ pour la collaboration à la recherche $(1,4 \%)$ et 6 pour la transmission des données de facturation à la caisse maladie $(1,0 \%)$, et 2 pour le recueil des données personnelles $(0,3 \%)$. Certaines limitations étaient multiples, expliquant un taux global supérieur à $100 \%$. La distribution des réponses en fonction des mois figure au tableau 2.

Chez 99 patients, une rencontre avec les infirmières chargées du recueil du consentement groupé n'a pas pu avoir lieu, pour de multiples raisons (absence d'attente avant la consultation, dû tant à la rapidité de l'anesthésiste qu'au retard du patient, ou indisponibilité de l'infirmière).

Les mêmes constatations que dans les services hospitaliers ont été recueillies sur l'accueil de la démarche et la compréhension de l'information, qui ne pouvait être donnée qu'une fois dans ce contexte.

\section{Discussion}

Cette double étude pilote a mis en évidence que l'élicitation d'un consentement groupé auprès de patients hospitalisés ou en consultation de pré-hospitalisation chirurgicale n'était possible que dans une proportion variable des cas (40\% en hospitalisation et $69 \%$ en pré-hospitalisation chirurgicale), et nécessitait un investissement important en temps et en personnel, pour une valeur ajoutée discutable, puisque $95 \%$ des patients qui ont rempli le document ont donné un consentement complet. Les limitations enregistrées touchent à des degrés divers les aspects de récolte de données cliniques, participation à la recherche ou à l'enseignement et transmission de données à l'assureur, de sorte qu'il est difficile d'envisager d'aborder tous ces sujets en bloc. A noter que ces rares limitations sont persistantes, puisqu'elles sont enregistrées dans le système d'information et apparaissent sur la carte administrative du patient à chacune de ses admissions.

Nos résultats montrent ainsi que la loi n'est pas applicable dans la mesure où d'autres lois qui doivent intervenir en amont ne sont pas appliquées non plus. C'est en particulier le cas des mesures de protection de la personne (curatelle ou tutelle) qui devraient être prises en principe dès que le patient perd sa capacité de discernement et qui ne le sont en fait qu'au moment où l'hospitalisation permet la mise en évidence de l'incapacité de discernement.

S'il est compréhensible qu'un pourcentage plus élevé de réponses ait été obtenu dans les disciplines chirurgicales, puisqu'un consentement spécifique est également requis pour que le patient puisse être soumis à l'intervention projetée, la situation dans les services de médecine n'est pas susceptible de s'améliorer. En effet, les patients deviennent de plus en plus âgés, souffrent de pathologies multiples, sont hospitalisés à de nombreuses reprises pour une maladie chronique qui s'aggrave progressivement. Il est important de souligner le plus faible taux de succès observé dans le recueil de consentement chez les patients déjà hospitalisés, l'état de santé du patient mobilisant toute son attention.

Par ailleurs, le recueil d'un consentement groupé à la consultation de pré-hospitalisation chirurgicale induit une certaine confusion chez les patients, puisque c'est également l'un des endroits où peut être recueilli leur consentement spécifique à l'intervention envisagée. La juxtaposition des deux procédures est difficile à comprendre, mais également à expliquer.

Ces résultats ne sont pas non plus susceptibles d'être améliorés si c'est le médecin assistant qui fournit ce type d'informations. En effet, les limitations du temps de travail récemment introduites ont obligé ces derniers à se recentrer sur les activités prioritaires de soins. Le consentement groupé n'en fait naturellement pas partie, à l'inverse du consentement spécifique nécessaire pour pratiquer des interventions. Il est donc peu probable que les médecins obtiennent de meilleurs résultats que les infirmières dédiées à cette tâche.

Une partie de l'activité d'enseignement et de recherche, indispensable à la relève et au progrès scientifique, une des raisons d'être des centres hospitaliers universitaires, se voit ainsi remise en question. Dans 
la majorité des cas, un consentement écrit ne peut pas être obtenu pour participer à des activités d'enseignement ou de recherche, et conserver et utiliser sous forme anonymisée ou codifiée des échantillons biologiques aux fins de recherche rétrospective et observationnelle, plutôt que de les détruire systématiquement. Il est difficile d'admettre que l'intérêt collectif puisse ainsi être mis en cause par des considérations techniques individuelles. Ceci est d'autant plus vrai que le taux d'acceptation par les patients est très élevé pour la participation à la recherche (de l'ordre de 97\%) [4], alors qu'il est plus faible pour la participation à l'enseignement, surtout en gynécologie ambulatoire (jusqu'à 30\% seulement) [5]. Cette limitation ne saurait naturellement pas concerner les protocoles de recherche prospectifs, qui nécessitent de toute manière un consentement spécifique du patient, et dont le principe n'est pas remis en question.

Plus préoccupant est le refus et le manque d'intérêt des patients pour cette démarche. Il reflète le fait qu'un effort particulier a été déployé ces dernières années pour fournir des informations aux patients, à leur demande, et qu'actuellement ils s'estiment submergés par l'information fournie, dans la mesure où leur préoccupation est avant tout centrée sur l'obtention des soins. Dans ce domaine aussi, un équilibre raisonnable doit pouvoir être obtenu, plutôt que la satisfaction complète d'un idéal technocratique et juridique.

Notre étude est naturellement limitée dans la mesure où elle s'est déroulée pendant une période de trois mois, sur deux services et une consultation seulement d'un seul centre hospitalier universitaire. De plus, elle dépend d'un contexte particulier, parce que la Loi sur la Santé Publique vaudoise contient des dispositions qui ne se rencontrent pas nécessairement dans les autres lois cantonales. La généralisation de ces résultats à d'autres systèmes hospitaliers ne peut s'effectuer sans précautions. Toutefois, les résultats étaient stables au cours des 3 mois, et sont donc interprétables.

Pour l'ensemble de notre établissement, la généralisation du système utilisé durant cette phase test nécessiterait l'engagement de 18 infirmières à plein temps, ce qui n'est pas envisageable à une période où les ressources du système de santé sont limitées et où la loi nous prescrit d'effectuer des prestations «efficaces appropriées et économiques» (LAMal art 32). Aucune de ces trois conditions n'est remplie dans cette démarche. Il semble ainsi légitime de se demander si la nécessité d'obtenir un consentement écrit pour ce genre de sujet (et non sur le traitement proposé au patient ou la participation active à la recherche) ne constitue pas un cas de formalisme juridique excessif.

Une alternative serait d'impliquer une politique de désaccord explicite, c'est-à-dire d'informer les patients et la population par voix de presse, par affichage dans l'établissement, et de mettre à disposition une ou deux personnes de contact pour répondre aux éventuelles questions et saisir dans le système informatique les refus et limitations de consentement exprimés par les patients. Cette solution aurait l'avantage d'être pragmatique, économique, mais ne respecterait pas la loi à la lettre. Cette alternative fera prochainement l'objet d'une troisième phase test d'un an au CHUV.

D'autres solutions doivent donc être explorées pour satisfaire les exigences légales actuelles. Ce consentement groupé pourrait par exemple être recueilli en amont, au moment où le patient souscrit une assurance maladie, et être inclus dans un document personnel de l'assuré de type carte de santé.

Cette expérience doit également être un signe d'alerte pour le législateur, attirant son attention sur les difficultés d'application des lois - parfois contradictoires - qui pourraient s'avérer encore plus contraignantes que celles qui ont été évoquées ici et fragiliser l'activité de recherche, comme par exemple la loi fédérale sur la recherche sur l'être humain, actuellement soumise au Parlement.

\section{Références}

1 Code Pénal Suisse du 21 décembre 1937 (www.admin.ch/ch/f/rs/c311_0.html).

2 Loi sur la santé publique vaudoise du 29 mai 1985 (www.rsv-fic.vd.ch/print-800.01.pdf).

3 LAMal du 18 mars 1984 (www.admin.ch/ch/f/rs/c832_10.html).

4 Vermeulen E, Schmidt MK, Aaronson NK, Kuenen M, van der Valk P, Sietses $\mathrm{C}$ et al. Opt-out plus, the patients' choice: preferences of cancer patients concerning information and consent regimen for future research with biological samples archived in the context of treatment. J Clin Pathol. 2009;62:275-278.

5 Mavis B, Vasilenko P, Schnuth R, Marshall J, Jeffs MC. Medical students' involvement in outpatient clinical encounters: a survey of patients and their obstetricians-gynecologists. Acad Medicine. 2006;81:290-296. 


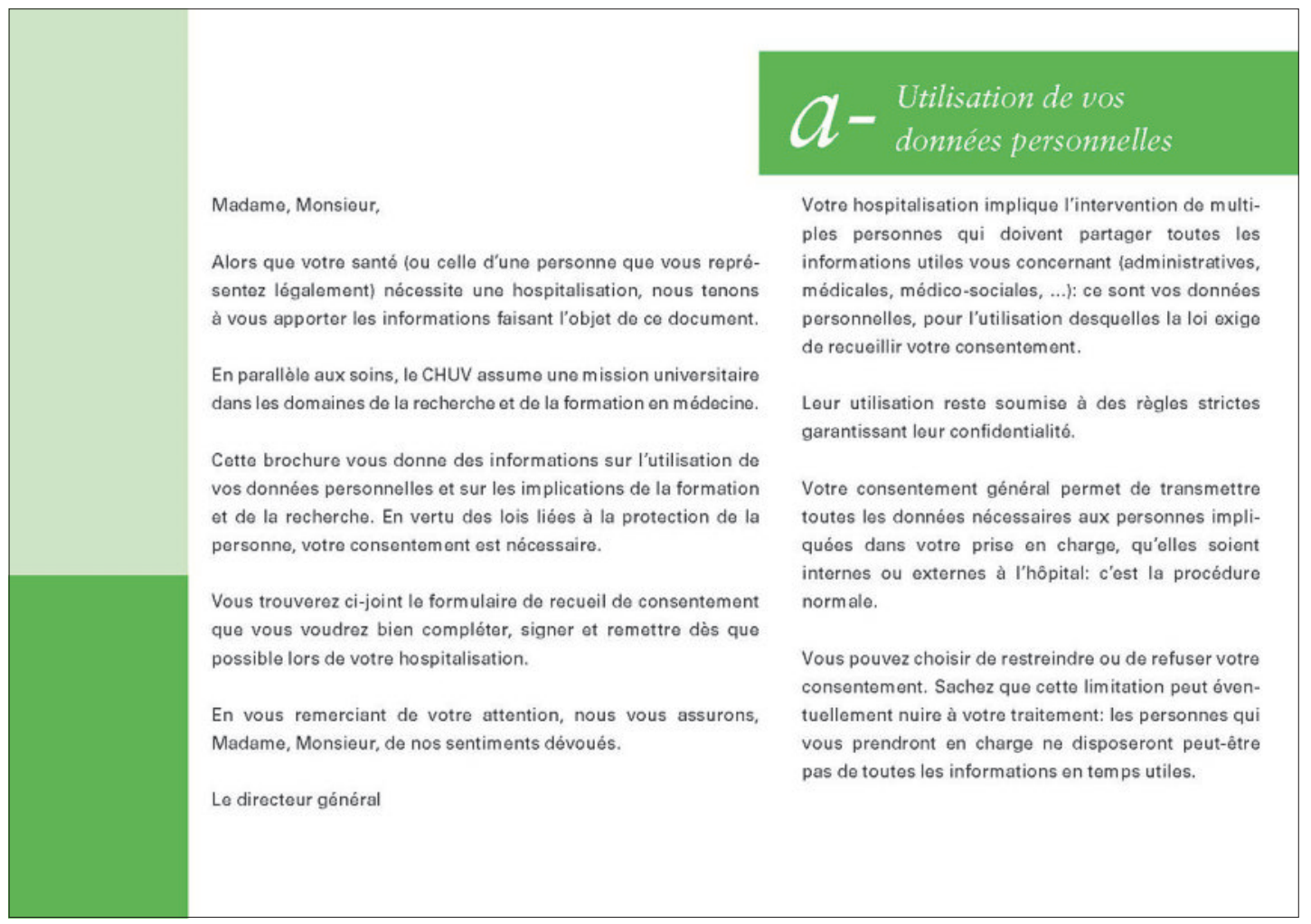

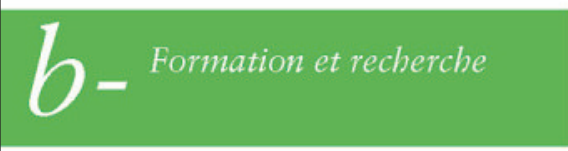

Le CHUV, hôpital universitaire, a aussi les missions de formation ot de recherche médicale. Cos missions s'accomplissent aussi dans le respect de la dignité et de la vie privée du patient.

Certaines personnes, parmi toutes les catégories professionnelles impliquées dans votre prise en charge, peuvent être en formation. Elles sont supervisées par des profossionnels compétents qui s'assurent de la qualité de leurs prestations.

Pour autant que son état le permette, le patient peut être sollicité pour apporter son concours à la formation des professionnels de la santé. Il est ainsi habituel que les visites médicales aient lieu en équipe; de ce fait, le patient peut se voir entouré de plusieurs personnes qui l'examinent. Vous pouvez le refuser, si cela vous expose à des désagréments importants.

A des fins d'enseignement, il est possible que l'on sollicite votre accord pour que des interventions soient filmées ou qu'olles fassent l'objet de publication scientifique. Dans ce cas, l'identité du patient n'est pas révélée et il n'est pas reconnaissable.
La recherche obéit aussi aux règles spécifiques on vigueur, notamment éthiques.

Le traitement du patient peut nécessiter des prélèvements de sang ou de tissus. Après examen, ces prélèvements sont soit détruits soit conservés pour d'éventuels examens complémentaires ultérieurs ou conservés dans un but de recherche. Sauf opposition de votre part, les données et prélèvements biologiques recueillis lors de votre séjour seront conservés et pourront être utilisés dans un but de recherche médicale ou d'amélioration des traitements. Dans la mesure du possible, les chercheurs travaillent sur des données et des prélèvements anonymisés ou codifiés.

Un consentement spécifique de votre part demeurera nécessaire pour requérir votre participation active à une recherche ou pour utiliser vos données ou prélèvements biologiques de manière personnalisóe. Dans ces cas, votre consentement sera recueilli apròs une information adéquate et vous conserverez le droit de le retirer en tout temps, sans aucune incidence sur l'attention et les soins qui vous seront prodigués.

\section{Registre vaudois des tumeurs}

Le Registre vaudois des tumeurs contribue à la lutte contre le cancer par la recherche dans les domaines de la prévention, du dépistage précoce et des causes de la maladie. Les diagnostics des tumeurs sont transmis au Registre qui est également soumis aux règles garantissant la confidentialité des données.

Cette transmission au Registre est autorisée par la loi, mais le patient qui le souhaite peut s'y opposer. Pour cela, il doit communiquer son opposition au médecin de I'hôpital. 
C- Facturation de votre hospi-

Depuis 2002, la facturation des hôpitaux vaudois se fonde sur un tarifforfaitaire par maladie, appelé APDRG (All Patients Diagnosis Related Groups), littéralement "groupes de diagnostics similairesn.

Les frais d'hospitalisation sont en principe payés directement par votre assurance maladie ou accidents, sous réserve de votre participation légale.

A la fin de votre hospitalisation, la facture est envoyée à votre assurance avec le code APDRG, mais sans l'in titulé de la maladie. Par exemple, le code APDRG 167 correspond au traitement d'une appendicite. Ce code permet à l'assurance de se déterminer sur le paiement de la facture et constitue une obligation légale. En son absence, l'assurance peut refuser de payer la facture et vous seriez alors tenu de le faire.
Si vous ne voulez pas que cette information soit communiquée à l'administration de votre assurance, vous pouvez demander à ce qu'elle soit transmise au médecinconseil de l'assurance qui est tenu par le secret professionnel. Dans ce cas, veuillez cocher la case correspondant au souhait de limiter les procédures habituelles sur le formulaire de consentement. Sinon, nous considérerons que vous acceptez que vos factures soient envoyées à l'administration de votre assurance maladie ou accidents.

Pour information complémentaire à ce sujet, vous pouvez prendre contact avec la permanence de notre service administratif, au téléphone 0213145757 .

Direction générale Hospices-CHUV Unité des affaires juridiques et éthiques Bugnon 46 Fax (a)

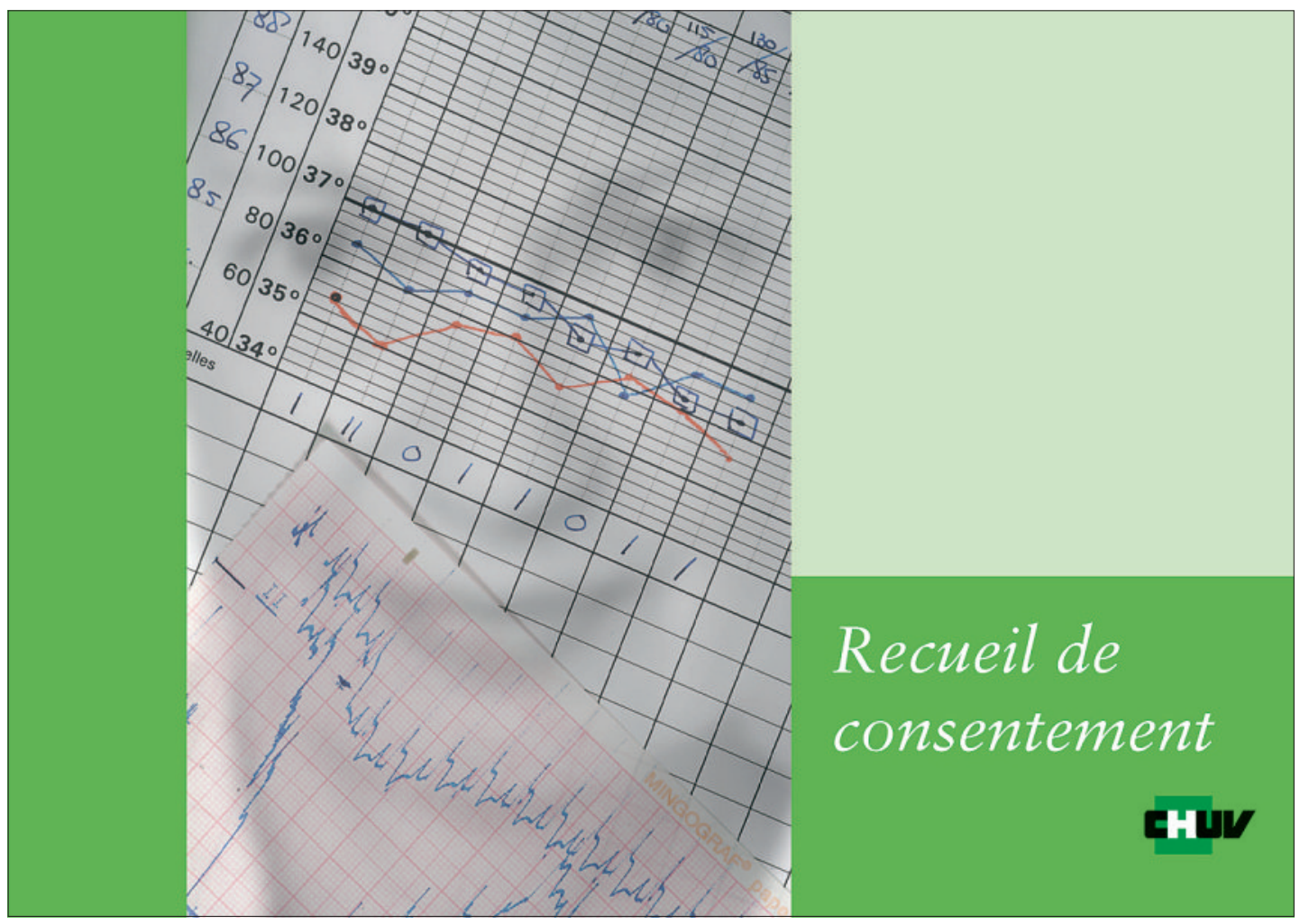

